\title{
A Machine Learning-based Damage Prediction Techniques for Structural Health Monitoring
}

\author{
M Vishnu Vardhana Rao', Aparna Chaparala ${ }^{2}$ \\ ${ }^{1}$ Research scholar, Department of CSE, Acharya Nagarjuna University, Guntur-522510, Andhra Pradesh \\ .2 Professor, Department of CSE, RVR\&JC College of Engineering, Guntur-522019, Andhra Pradesh. \\ mvvrao.mca31@gmail.com
}

\begin{abstract}
Article History: Received: 11 January 2021; Accepted: 27 February 2021; Published online: 5 April 2021 Abstract: Nowadays, the Structural Building Health Damage Monitoring System (SBHDMS) is a crucial technology for predicting the civil building structures' health. SBHDMS contains abnormal changes in the buildings in terms of damage levels. Natural Disasters like Earthquakes, Floods, and cyclones affect the unusual changes in the buildings. If the building undergoes any natural disaster, the sensors capture the vibration data or change the buildings' structure. Due to the vibration data, these unusual changes can be analyzed. Here sensors or Machine Learning based Building Damage Prediction (MLBDP) are used for capturing and collecting the vibration data. This paper proposes a Novel Rough Set based Artificial Neural Network with Support Vector Machine (RAS) metaheuristic method. RAS method is used to predict the damaged building's vibration data levels captured by the sensors. For the feature reduction subset, we use one of the essential preprocessing method called the Rough set theory (RST) strategy. RAS has two contributions. The first one is the Support Vector Machine (SVM) classification method used for identifying the structures of the buildings. The artificial Neural Network (ANN) method used to predict the buildings' damage levels is the second contribution. The proposed method (RAS) is accurately predicting the conditions of the construction building structure and predicting the damage levels, without human intervention. Comparing the results states that the proposed method accuracy is better than SVM's classification methods, ANN. The prediction analysis depicts that the RAS method can effectively detect the damage levels.
\end{abstract}

Keywords: Machine Learning, Structural Health Monitoring, Data Mining, Rough set theory, Machine Learning based Building Damage Prediction (MLBDP).

\section{Introduction}

In the 1960s, a local assessment system is implementedfor identifying the damage levels in civil infrastructures called as Structural Health Monitoring (SHM) or Structural Strength Monitoring System (SSMS). Typically indicates the offline assessments such as visual inspection, Gamma, and X- rays. Building structures are helpless against impacts like natural disasters, earthquakes, and typhoons. Regular inspection of the building gives damage identification (DID). However, it is impossible because of the time-consuming factor. Mitigate the regular inspection cost, and increase public safety, need a robust procedure for diagnostic automatically called Structural Strength Monitoring System (SSMS). It used to estimate the lifetime (Strength) of the buildings. Input to the SSMS is raw data obtained from a different kind of MLBDP. It is like Accelerometer, Thermometers, Hygro-meters, and Extenso-meters that mounted on buildings or bridges. The first requirement for SSMS is building structure observations overtime or a long time through the MLBDP deployed along with the whole building structure. It uses the synchronized data, correlated with the data coming from different MLBDP.

By early predication of the damages in the buildings is used for extending the strength (lifetime) of the building structures and increase public safety. The essential features are the structural parameters and structural performance of the buildings in case of natural disasters. The parameters like signals, displacement, velocity, and acceleration generally used for monitoring the strength of the building. Forces derived from acceleration measured by accelerometer and the derivation gives the displacement of the building structures. The Velocity variation reflects the damage of the building structure directly [1][2]. So identification of system parameters of buildings is essential for the structural monitoring or the damage detection of buildings. The parameters are having the number of properties in terms of physical and dynamical. Mass, stiffness, damping coefficients, number of stages, vibration states are the physical properties and natural frequencies, and mode shapes are the modal properties. Due to the impact of natural effects, there are abnormal changes in the structural parameters or changes in the building structures.It indicate that the structural damages presented.

The simplest way to measure the acceleration is by using accelerometers installed inside the buildings. However, the advantage is that accelerometers need not any specific position in the buildings. Using accelerometers to find acceleration and Velocity (numerical integration) and displacement [3]. For estimating the velocity and displacement used the numerical integration of the accelerometers [4].The data-Based method does not recognize the attributes of the building structures but used for identifying the displacement of the building structure. At the same time, so many reviews use the Data-Based methods [5] [6].

The proposed method uses the vibration data for predicting the damage levels of the building. Using Sensorscapture the vibration data in case of natural disasters like earthquakes, floods, cyclones, changes in the 
environmental conditions. The sensors mounted on each floor of the buildings. Most predictive applications have the numerical integration of the signals having the noise. So use some essential preprocessing techniques like Rough set theory, cancellation of offset, and high pass filtering techniques joined for solving the problem. The changes in the status of the faults indicate the changes in the environment. First, we find the feature reduction subset of the dataset; for that, the RST is used as a preprocessing method and applied to the dataset. This way, the missing values, duplicate values, not suitable attributes in the dataset reduced to derived attributes used as input to the one of the classification method SVM.

SVM method gives the building structure classification and ANN classification method used for finding the damage levels of the buildings. Comparisons prove that the accuracy levels of the classification of the proposed hybrid method greatly enhanced than individual classification methods. The contributions include the hybrid methods as a feature reduction subset, or dimensionality reduction made using RST strategy (pre-processing). The derived attributes from the dataset used for the classification of building structures and damage levels of the buildings using SVM and ANN. The hybrid method uses the vibration data for estimating the damage levels in the structures.

The organization of the paper as follows, in the next sections, explain the related work. In section three, discuss the methodology of SSMS and its building models. In section four, explain the fault analysis. In section five, discourse the novel method RAS. Next, explain the result analysis. Finally, stop with a conclusion and future work.

\section{Related work}

In general, Damage defined as "voluntary or involuntary modificationshosted into a system which is harmful, emotional impact on the existing or upcoming efficiency ofthe particular system. The damage can be either natural or human-made. The idea of damage is not important without assessment between two different states of the system. C. R. Farrar and K Worden [7] says that the definition of damage is "alteration in material properties or changes in geometrical positions or locations. It contains boundary circumstances and connectivity of the system, that badly disturbs the system accuracy". SSMS systems need analysis information in order to go beyond the mere damage detection task. The SSMS has been used to evaluate the state of the structure at different levels of abstraction.

Now the damaging impact on a structure can be classified as linear or Non-linear. Rytter, 1993 [8] defines the four levels of damages, and these levels are used for damage identification (DID) in the structures as follows.

Level 1: Estimate the presence or absence of the damages in the structure. This level called a Detection level.

Level 2: The presence or absence of the damage is determined and also find the location of the damage (geometric location). This level is called Location.

Level 3: It specifies the dimension of damage (size) and its strictness (severity). This level is called severity.

Level 4: Predicate the leftover service life of the building (structure). This level is called a prediction.

The following are some of the literature reviews focus on the DID Levels one, two, and threei.e., Estimation of the damage existence, position, and strictness. Doubling et al. [9] reviewed on vibration-based damage identification methods in 1997. Sohn et al. [10] up to 2001 and Carden and Fanning [11] reviewed applications. Wang and Chan [12] reviewed the recent development in statistical damage detection. Fan and Qiao [13] reviewed modal parameter-based DID methods and the study of five algorithms. Moughty and Casas [14] reviewed the deployment of unconventional computational methods, Das et al. [15] reviewed on variant vibration-based DID methods. However, they did not include level4 (predicate the leftover service life of the buildings and decision making) as a research topic. Therefore, Xuan Kong, Chun-Sheng Cai, and Jiexuan $\mathrm{Hu}[16]$ develop the state-of-the-art on the framework of vibration-based DID in variant levels are the damage existence, damage position, damage sternness, leftover life prediction, and decision making. The identification of damages in structures by observing the changes in dynamic properties such as modal strain energy [17-22], natural frequencies or mode shapes [23-32], frequency response functions [33, 34],flexibility-based displacement [3537] and modal curvature [38]. Now by observing the changes in modal strain energy, identify the deficiencies in the structures using the very favorable vibration-based method is the Damage Index Method (DIM). In 1992 Stubbs et al. implements the DIM, utilizes the absolute changes in modal strain energy earlier and later damage to recognize defects. Due to significance, damage identification becomes a pattern recognition problem. The presence of noise in the data gives different results. Artificial neural networks (ANN) are a robust tool for handling pattern recognition problems and noise data very successfully. DackerMann et al. [39] develop a dependable and robust procedure to recognize the damage in beam structures using the DIM hybrid with ANNs. At the same time, Dackermann, Samali et al. [40] utilizing the Neural Network Ensembles (NNE). In 1990, Hansen \& Salamon industrialized a methodcalled Neural Network Ensemble. This method is a learning model 
where a pool of neural nets a hierarchy for identifying the damages based onvariations in Modal strain Energy applied to simulate field-testing conditions. White Gaussian noise is supplementary to statistical data, and questions with a restricted number of sensors integrated. This paper presents a Vibration-Based Damage Identification Method (VBDIM), which develops the DIM combination with NNE to identify the position and severity of single damage.Adams et al. [41] developed an approach and worked on a 1D component. This method determines the detection of damage using the longitudinal vibrations of natural frequencies.U. Dackermann, J. Li \& B. Samali defines the technique for damage identification. This technique identifies the boundary conditions of the structure using the output, only scalar measurements of the structure. A DI is expressed based on output-only acceleration reaction dimensions from ambient floor shaking. Measurements in unification with One pre-processing method called PCA and one classification method ANN techniques. PCA and ANNs were effectively functional to a mathematical and experimental 2-story framed structure [42]. From this analysis, it created the suggested damage detection algorithm capable of accurately and reliably detecting damage using only measured floor vibration responses from impact loading (Dackermann, Li \& Samali 2010) and (Samali, Dackermann \& Li 2012). Doebling et al. (1996) give a review of Modal-Based Damage Detection Methods. Carden \& Fanning (2004) presents a comprehensive review of the literature on Modal Based Damage Detection Methods

A comprehensive literature review on model-based damage detection methods found in (1996).

Kim and Stubbs et al. [43] developed the Single Damage Indicator (SDI) method. It works on the crack location model, crack size model for estimating the zone, quality of the cracks in the beam-type building structures. This estimation predicted using the changes in both modal energy and natural frequencies. Result analysis can be done using a small number of natural frequencies and applied on the 16 test beams.

Tripathy [44] developed the genetic algorithm (GA) to decide and evaluate the structural damage. Using the changes in the building parameters and also find the range of damaged building structures using the changes in natural frequencies. GA applies to little cracks in the buildings of beam type building structures.

Pawar and Ganguli [45] develop the genetic fuzzy system approach to find the matrix crack and the changes in natural frequencies. The genetic fuzzy system method worked on thin-walled composite building structures. Based on the changes in structural parameters such as damping and stiffness coefficients, the status of the damage levels and strength of the building changes.

Gudmundson et al. [46] developed the energy-based perturbation approach and worked on damaged building structures. This method determines the range of damaged building structures using the changes in the resonance frequencies. However, the major problem is the loss of mass and stiffness of the Buildings.

Kasper et al. [47] developed an approach and worked cracked symmetric uniform beam. This method determines the range of damaged building structures using the wavenumber shift and frequency shift.

Morassi et al. [48] developed the Euler-Bernoulli beam theory. This method worked on the beam type building structures having the crakes. In this model, to estimate the cracks of the building structures using the comparison between the frequency of the cracked beam-type building structure. That means determining the cracks of the buildings done by observing the changes in the frequency comparison between potential energy and frequency sensitivity stored undamaged beam. However, the problem is that expressions are valid only for a small defect.

E. Lughofer et al. proposed that the confinement in assets of sensors dependent on the IEEE 802.15.4 standard. This critical challenge contemplated, which is how to adjust IP stacks on WSN effectively. Distinctive arrangements have been created as of late to predict the building structure's strengths. In this proposed work, the estimate of the fault status done by using building parameters like the natural frequencies and mode shapes parameters used as input to the neural network for fault identification. To confirm the accuracy and efficiency of the proposed method is suitable using the Mathematical patterns on buildings.

Chinchalkar et al. [49] developed a Finite element-based numerical approach for estimating the damage and simulate the semi-analytical approach by using the Frobenius method. The benefits are relatively simple, easy to apply to the buildings with different boundary conditions, and it does not require multiply precision computation. Nevertheless, the problem is only applicable to the beam type buildings with little cracks. Morassi and Rollo [50] developed a comprehensive method for prediction of 2 cracks having the same severity in the simple beam based on the identical changes in the first three natural frequencies (Flexural vibrations). Advantages are the Cracks with different severity in two sets of different locations. However, the disadvantage is that the method applies to a simple and small beam-type building structure with little cracks. 
H. Hothu et al. demonstrate the performance of SVM for tracingthe damage in the structures using two sensors and the first three natural frequencies [51]. D. K. Srivastava, K. S. Patnaik, L. Bhambhu [77] developed the hybrid method for classification problem called A Rough - SVM Approach for Data Classification. This paper informs that the overview of the Rough -SVM Approach established on the hybridization of RSES (Rough Set Exploration System) and SVM. RST is effectively used in feature selection and then applied to SVM for improved classification results. i.e., SVM classification system hybrid with the RST. RST act as a pre-processing step. Now the given training set, at first, separate them if the values of the feature are continuous and get a negligible attribute subset that fully defines all ideas by feature reduction[76][52]. Building an SV classifier and estimate the decision function. When given test sets, we moderate the equivalent features and place into the SVM classification system, then we can obtain the testing outcome. Rough Set Exploration System (RSES version 2.2) and LIBSVM-2.85 have been used to bring out experiments on the heart data set [53]. A Rough-SVM, which creates countless use of the benefits of SVM's better simplification presentation and RST, is successfully dealing with vagueness and ambiguity statistics. Classification accuracy greater than before by $4.29 \%$. We can notice that the classification accuracy using Rough-SVM is considerable better than the general SVM and general RST method.

Zhong Zuowei1 Mu Lili [54] develop the Integrated Methodology of Rough Set Theory and Artificial Neural Network for Safety Assessment on Construction Sites. A hybrid intelligent system combining rough set approach and neural network Hybrid method means combining the RST and ANN. RST used for feature selection. Most of the datasets having redundant attributes. Due to this, the processing time increased. So in this method, RST is used as a pre-processing step for removing the redundant attributes without loss of information and also creates some rules for outstanding prediction accuracy. Automatically the processing time is reduced and improves the performance prediction capability by ANN. The outcome of RST reduced the information table, and then reduced information is used to develop classification rules and train neural networks to infer appropriate parameters. The efficient statistics structure by RST provides for NN training. However, the problem is Object noise. NN technology separates the input data, non-sensitive with noise, and got a high degree of prediction accuracy. This paper reports the loose coupling hybrid system joining RST and ANN.The Structure of Hybrid Models contains the following steps Data Discretization, Attribute Reduction, Object Reduction, and Rule Generation.The Rough Set Data Analysis handles all steps. The RST used for reducing the input samples of the $\mathrm{NN}$ and estimate the association between the features to make straightforward the structure of NN. The input to the data discretization and data reduction is a construction information system. Then the outcome data is the target of NN training. In this paper, the analysis of the experiment performed with ROSETTA software, which is the knowledge discovery Decision (KDD). RS-ANN used a sigmoid function for activation and backpropagation algorithm for learning. We use STATISTICA6.0 software for a neural network to achieve the algorithm, as mentioned above. The maximal relative error in the RS-ANN model is only $4.21 \%$, and the present analysis applies only at the attributes reduction and back-propagation neural network. The advantage of this hybrid approach is that a reduced input required for neural network modeling. The effectiveness of our methodology verified with the empirical study that compared the neural network approach with the hybrid approach.

Several optimization techniques are used to solve the structural damage detection problems, such as ant colony optimization (ACO) [55], particle swarm optimization (PSO) [56-59], artificial bee colony optimization (ABC)[60], genetic algorithms (GA) [61-65], neural networks (NN) [66, 67]. Forstner et al. [68] uses the classification methods for finding the modal constraints, such as the damping coefficients, stiffens of the buildings, building structure's natural frequencies, and the vibration strength. This paper recognizes building structure features by compares the alterations or variations from the standard output and the actual output using Data-Mining techniques such as SVM and ANN.

\section{Structural strength monitoring system (ssms)}

The real scrutiny does not offer an effective way of formative structural degradation. So consequently, a method is crucial for investigating the damage structures and levels of the building. SSMS is one of the investigating methods and regularly monitoring the building structures. The permanent structural monitoring system is a predictable procedure for observing the changes in the structural parameters. These changes give the status of the building structure's health. Frangopol et al., 2011; Housner et al., 1997 and Worden et al., 2007; defines the Non-Destructive Method (NDM) for predicting the degradation states of the building structures using regular inspection. However, the critical issues to be considered for developing the method is to improve the accuracy, update, and integrity of the building structure data. Now the SSMS contains some steps for getting better accuracy.

The following four steps needed for finding the strength of the buildings. 
1. Operational Evaluation: It says that SHM related with the parameters like economic, public life protection, environmental conditions for operations

2. Data Gaining: It gives data preprocessing used for feature extraction

3. Feature extraction: This step describes the properties of the preprocessed vibration signals (output of the above step). It gives the best signal for finding the damage remaining signals eliminated using any transformation techniques. Usually, the features are stiffness, mass, and displacement.

4. Model for feature classification: The output of the above step implements any classification algorithms.

The dynamic building parameters, such as velocity, displacement, stuffiness, and damping ration (vibration data) changes from time to time. Velocity, Displacement plays a vital role in the diagnosis of the fault in terms of building structures and damage levels of the buildings in case of natural disasters like earthquakes. For predicting the structural health of the civil buildings, the primary data is vibration data. The accelerometer and MLBDP are used for capture, gathered the parameters of the buildings when extra forces applied to the buildings. The vibration data used to detect defects in the building.Moschus et al. [69] specified dynamic features like displacement. So the Health monitoring system uses the vibration data. The vibration data used as input to SSMS for predicting the status of the buildings and also assess the place, possible levels of the damages in the buildings. Approximately SSMS uses onlookers to evaluate the frequency and location using the acceleration signal [70][71].

Identify building parameters using data mining techniques SVM and ANN by comparing scientific products and original products. Localization and authentication issues more appropriately solved with the appropriate combination of global dynamic and EMI techniques with artificial neural networks (ANNs). Therefore, the integration of these strategies leads to a more efficient SSMS. We need a building model for that. This model is used to detect faults in buildings by comparing vibration data with the model. SSMS becomes a pattern recognition problem.

\subsection{Building model:}

The main aim of SSMS is to determine the damage in the Buildings. For that, SSMS uses the dynamic responses (vibration data of the buildings) of the Buildings and regular monitoring of the Buildings. By applying the external force or ambient force or any natural hazards, the sensors capturesthe changes in geometrical positions of the Building. That means the sensors having the dynamic responses of the building.Sensors gathers the vibration data. So we need one basic model for doing all these things called the Building model. However, this model demonstration in what way to observe the building and classify the vibrant reactions used for finding the damage of the buildings. Then these are used for SSMS finding the damage of the buildings. Building models assist us in illustrating the alteration among the model- and data-based SSMs. Mass p, Damping d, and Stiffness $s$ are the three essential components for the single-degree-of-freedom (SDOF) building structure. The stiffness $s$ can be modeled by either elastic or inelastic (linear or nonlinear). Linear means SDOF, and all other buildings are nonlinear [72]. The mass $p$ generally measured as a constant. For getting the dynamic response from the building by applying the ambient force $f$ to the building. This vibration data (dynamic response) contains the following three components, namely $\mathrm{x}$ "acceleration, $x$ is displacement, and velocity is $\mathrm{x}$ '. Now the equation (1) is Newton's motion equation of an SDOF (linear or elastic) system visible to an ambient force $\mathrm{f}$ is

$$
p x^{\prime \prime}+d x^{\prime}+s x=f \text {. }
$$

Then the following equation (2) used for finding the force F, in terms of acceleration, displacement, and velocity of linear construction with $n$ Degree Of Freedom (NDOF, $n$-floor building structures) are

$$
P x^{\prime \prime}(\mathrm{t})+D \mathrm{x}^{\prime}(\mathrm{t})+S \mathrm{x}(\mathrm{t})=\mathrm{F}
$$

Where, $\mathrm{P}, \mathrm{D}, \mathrm{S} \in \mathrm{R}^{\mathrm{n} \times \mathrm{n}}$ the stiffness matrices, damping, and mass, respectively. $\mathrm{x}^{\prime \prime}(t), \mathrm{x}^{\prime}(t)$, and $\mathrm{x}(t) \in \mathrm{R}^{\mathrm{n} \times 1}$ the acceleration, velocity, and displacement vectors, respectively, and $\mathrm{F}$ is the external force vector.

The health of the building structures stated using the following three signals, namely acceleration, velocity, and displacement $\mathrm{x}^{\prime}(t)$, $\mathrm{x}^{\prime}(t)$, and $\mathrm{x}(t)$. The first component directly related to Force $\mathrm{F}$, whereas the other twocomponent are much closed with the faults diagnosis of the building structures. Here, displacementx $(t)$, velocity $\mathrm{x}^{\prime}(t)$ used for observing the health status of fundamental building structures.

The equation (3) is a nonlinear function for stiffness component in the case of nonlinear (inelastic). It used for modeling the stiffness component. The following nonlinear function is the modeled used for the stiffness component is inelastic.

$$
p x^{\prime \prime}+d x^{\prime}+f_{b}\left(x, x^{\prime}\right)=-p x_{g}^{\prime \prime}
$$

Where $\mathrm{x}^{\mathrm{n}}$ acceleration of ground, $f_{b}\left(x, x^{\prime}\right)$ is the force in the form of nonlinear function [73].

$$
f_{b}\left(x, x^{\prime}\right)=\tilde{\alpha} k x+(1-\tilde{\alpha}) f_{r} \tilde{\eta} \mathrm{k}
$$




$$
\dot{f}_{r}=\frac{\tilde{\delta} x^{\prime}-\tilde{v}\left(\tilde{\gamma} x^{\prime}\left|f_{r}\right|^{\tilde{n}}+\tilde{\beta}\left|x^{\prime}\right|\left|f_{r}\right|^{\tilde{n}-1} f_{r}\right)}{\tilde{\eta}}
$$

Where $\dot{f}_{r}$ introduces inelasticand $\tilde{\eta}, \tilde{\beta}, \tilde{\delta}, \tilde{v}, \tilde{\gamma}$ and $\tilde{n}$ the controlling parameters affect the shape of the system, $\tilde{\delta}, \tilde{\alpha}, \tilde{\eta}$ and $k$ is the control variable for the initial curvature stiffness. Now $x^{\prime \prime}(t)$ using accelerometers assessed only acceleration.

W.Yu et al. [74] developed a novel to estimate the velocity and displacement using numerical integration. By integrating, acceleration obtain the velocity $x^{\prime}(\mathrm{t})$ and displacement $x(\mathrm{t})$.

$$
x^{\prime}(\mathrm{t})=\int_{0}^{t} x^{\prime \prime}(\tau) \mathrm{d} \tau+\mathrm{x}^{\prime}(0) x(\mathrm{t})=\int_{0}^{t} \int_{0}^{\tau} x^{\prime \prime}(\tau) \mathrm{d} \tau d t+\mathrm{x}^{\prime}(0) t+x(0)
$$

Now applying the numerical interpolation for numerical integration for acceleration using the Eq. (6)

$$
\int_{t_{0}}^{t_{n}} x^{\prime \prime}(\mathrm{t}) \mathrm{dt} \approx \sum_{i=1}^{n}\left[\frac{x^{\prime \prime}(i-1)+x^{\prime \prime}(\mathrm{i})}{2}\right] \Delta_{t}
$$

The combination procedure is uncertain due to the low-frequency preference, and the origins are associated with the integrator. To eliminate the frequency mechanism done by using the high-pass filter with two poles. The below function shows that transfer function for two poles high filter is

$$
G(\mathrm{sd})=\frac{(s d)^{2}}{(s d)^{2}+\left(\frac{2}{\tau}\right) \mathrm{sd}+\frac{1}{\tau^{2}}}
$$

In the above function is the filter time constant, estimate the filter time constant Fast Fourier Transform (FFT) used. The cut-off frequency estimate, the frequency distribution of FFT $f_{c}=\frac{1}{2 \pi \tau}$ of the filter (7). The $f_{c}$ used to distribute the noise. The cut-off frequency $f_{c}$ nominates such that it shrinks the statistics data. Thus, those low-noise accelerometers are a good selection, since low cut-off frequency.

\subsection{Building Health Structure Monitoring:}

The sensor data contains statistical properties. Using these properties to estimate the damage levels. The mean $\bar{x}$, kurtosis $k u$, standard deviation $s d$, skewness $s w$ are the numerical instants of the statistical properties.

$$
\bar{x}=\frac{1}{n} \sum_{i=1}^{n} q_{i} s d=\sqrt{\frac{1}{n} \sum_{i=1}^{n}\left(\mathrm{q}_{i}-\overline{\mathrm{x}}\right)^{2}} s w=\frac{\frac{1}{n} \sum_{i=1}^{n}\left(\mathrm{q}_{i}-\overline{\mathrm{x}}\right)^{3}}{s d^{3}} k u=\frac{\frac{1}{n} \sum_{i=1}^{n}\left(\mathrm{q}_{i}-\overline{\mathrm{x}}\right)^{4}}{s d^{4}}-- \text { (8) }
$$

When the kurtosis $k u>\bar{x}$, here better responsiveness adjacent values. It disturbs, deviations of PDF (Probability density function), CPD (cumulative probability density) used for the prediction of the damage levels of the building structures. The central limit theorem says that the distribution of the sum of a random probability distribution or pattern that may be analyzed statistically. However, it predicted precisely that event variables frequently behave in a particular way or have a specific characteristic normal distribution. These are the regions to accept that the distribution of the base state is normal.The below figure 1 demonstrated how the frequency responses of the building structures represented. The occurs of damages in the buildings are represented by the changes in the peak values. When the damage level status increases, then the deviation of the Probability density function high with comparing normal distribution. When ambient force $\mathrm{f}$ applied to the building structure. 


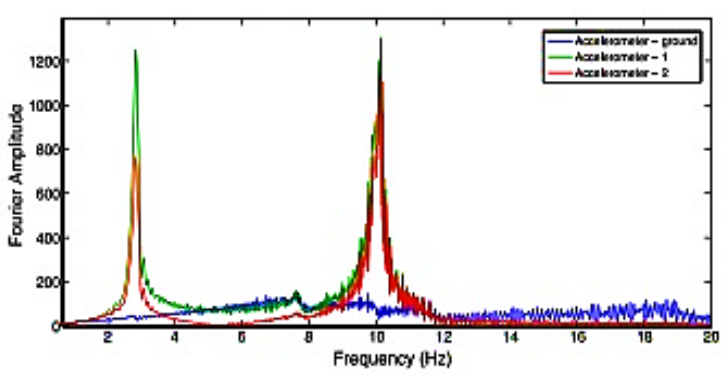

Figure 1. Frequency responses of the building structure.

\section{FAULTS ANALYSIS:}

The buildings mounted each floor with the $\mathrm{n}$ number of accelerometers, then building structure (1) produces vibrant responses at $s$ the time. Now the displacement is $x(\mathrm{~s})=\left[\mathrm{x}_{1}(\mathrm{~s}) \ldots \ldots \mathrm{x}_{n}(\mathrm{~s})\right]^{T}$. The velocity$x^{\prime}(\mathrm{s})$, acceleration- $\mathrm{X}^{\prime \prime}(\mathrm{s})$ for all $n$ floors. $\mathrm{x}_{0}(\mathrm{~s}), x_{0}^{\prime}(\mathrm{s})$ and $\mathrm{x}_{0}{ }^{\prime \prime}(\mathrm{s})$ are defined as signals on the base floor. In order to minimize the effect of the input force, for example, seismic excitation, we use the relative displacement and velocity

$$
x_{i, 1}(\mathrm{~s})=x_{i}(\mathrm{~s})-\mathrm{x}_{0}(\mathrm{~s}) x_{i, 2}(\mathrm{~s})=x_{i}^{\prime}(\mathrm{s})-\mathrm{x}_{0}^{\prime}(\mathrm{s}) \text {..(9) }
$$

Where $i=1 \ldots n ; s=1 \ldots t$; and tis the total data number. The mathematical combination of the acceleration is as follows:

Velocity $\mathrm{X}_{2}(\mathrm{~s})=\left[\mathrm{X}_{1,2}(\mathrm{~s}), \ldots \ldots . ., \mathrm{X}_{n, 2}(\mathrm{~s})\right] \in R^{n} \quad$ and $\quad \operatorname{displacement} \mathrm{X}_{1}(\mathrm{~s})=\left[\mathrm{X}_{1,1}(\mathrm{~s}), \ldots \ldots . ., \mathrm{X}_{n, 1}(\mathrm{~s})\right] \in R^{n}$. The damage diagnosis understood as

$$
a_{i 1} \leq\left|x_{i, 1}(\mathrm{~s})\right| \leq b_{i 1} c_{i 2} \leq\left|x_{i, 2}(\mathrm{~s})\right| \leq d_{i 2} . .(10)
$$

Where $a_{i 1}, b_{i 1} c_{i 2}$ and $d_{i 2}$ are earlier defined constants. The label $\left(x_{i, 1}(\mathrm{~s}), x_{i, 2}(\mathrm{~s})\right)$ is defined as +1 means no damage. Otherwise, it $\left(x_{i, 1}(\mathrm{~s}), x_{i, 2}(\mathrm{~s})\right)$ is -1. In Figure 2, they are marked as “o”, “+” [75].

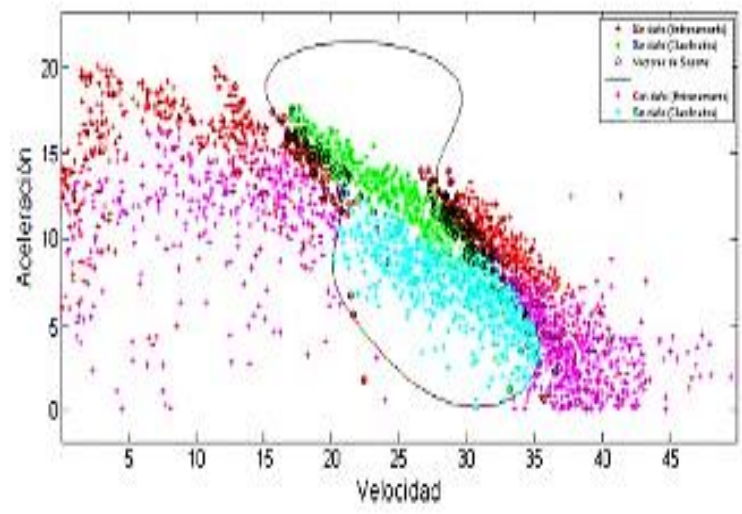

Figure 2. Damage and non-damage Data

\section{5. ras novel methodology}

The below figure 3 shows the flow chart for the prototype of the suggested technique. Now from standard repository, choose the best data set. Data Acquisition is a minor step, describe how to gather, store the needful information from the MLBDP, number of MLBDP that used, location of the MLBDP, filters for MLBDP. The collected data having the noise, low-frequency then in feature extraction step uses the filtering eliminates the noise. Also, use any suitable preprocessing methods to get the needful information for getting better results or accuracy. The feature extraction step is a significant step, defines the attributes of the preprocessed vibration signals. It is the superlative signal for finding the damage remaining signals eliminated using any transformation techniques. Usually, the features are stiffness, mass, and displacement. In the proposed method, we use the Rough set Theory (RST) for Feature Extraction or dimensionality reduction or attribute reduction or knowledge reduction. In the total dataset, $70 \%$ of data are used for training and remaining for testing. RST based selection of 
Features and parameters then send to the training of ANN/SVM. Now training completed, using the test dataset find the Trained ANN/ SVM with selected features. After that, get results like predicting the damage levels of the building structures, then the final output is Predicating the damage of the buildings. The selection and classification properties of the input attributes are optimized using a rigorous set-based approach. These characteristics, that is, the root mean square (RMS), the difference, slope, kurtosis, and generalized high order (up to ninth) pivotal moments used to distinguish between the standard and defective bearings.

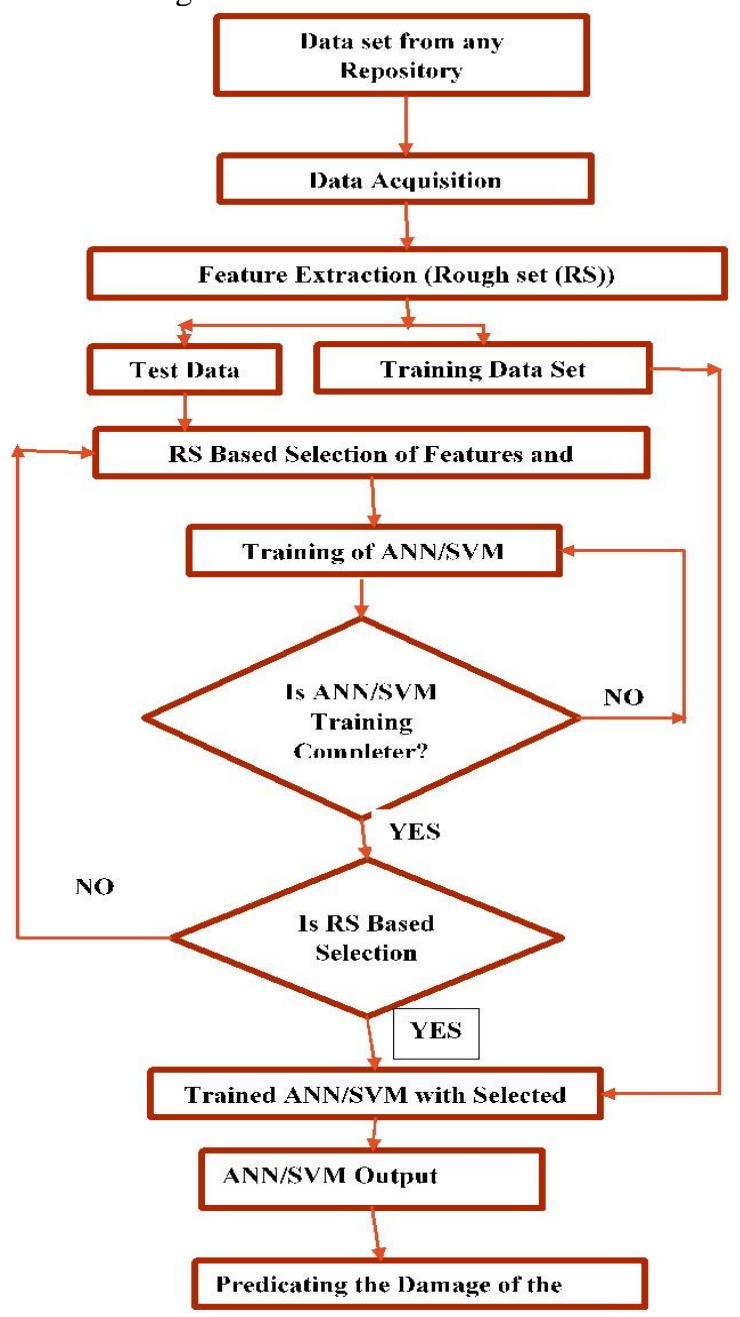

Figure 3: prototype model for the proposed method.

\subsection{RST, SVM, and ANN:}

The essential benefit of considering a rough set for reducing the features and selecting the attribute subset is the higher reduction in time complexity. The model of the rough set for attribute subset selection starts with a generic empty set and keeps on adding features from the existing set of attributes. While adding the new features, if the dependence degree increases, then the new feature is made a part of the subset. The termination point is the point where the addition of the new feature or attribute does not impact on the degree of dependency.

\begin{tabular}{l}
\hline Algorithm 1: Rough Set \\
\hline Step -1. Accumulate the \{conditional attributes $\}$ \\
Step -2. Accumulate the \{decision attributes $\}$ \\
Step -3. Consider an empty set for the final attribute \\
group \\
Step -4. For all attributes in the decision attributes set \\
a. Calculate the dependency degree change \\
b. If an increase in dependency degree i. \\
Include the attribute in the final attribute group \\
Step -5. Generate the final attribute set
\end{tabular}


Now the benefits of the RST method deploys in the proposed algorithm for selecting the higher accuracy and, at the same time, eliminates the redundancy by deploying the rough set algorithm. Because of the time required for training, the dataset increased due to redundancy attributes. So RST reduces the training processing time by eliminating the redundancy attributes. The variations in the parameters of the building's structure, damage detection viewed as a pattern recognition problem. The mapping derived from some parameters affects the change in the physical properties of a system to the health condition of a building structure. The pattern recognition process consists of data acquisition, feature extraction, and classification or description. Pattern recognition into statistical and structural methods based on the approach used for classification or description. Statistical pattern recognition methods based on some statistical measures derived from quantitative properties of the input data. Machine learning techniques that try to learn a function to map the input class with the output class (labels) in the training dataset. This classifier margin (plane) is the superlative judgment boundary between the two sets of data. Now identification of damages, location of the damages become the classification problem. This problem solved by using SVMs classifier in SSMS. Attributes derived from the vibration data. The vibration data gathered from accelerometers mounted on each floor of the buildings used by binary classification methods. The SVM technique to generate the final margin classifier between the data sets derived from the damaged and the whole building structure.

Both the undamaged and damaged classes of data than used in the SVM technique as training data. The training dataset, the best possible hyperplane is then computed between these two classes of data. The evaluation of the best hyperplane involves choosing the parameters of the SVM, the optimum percent reduction of the whole training class, then solving the SVM optimization problem. The health condition of a building structure can then be predicted by finding on what side of the SVM hyperplane does a test point derived from the building structure lie. It expected that the trained SVM would be able to detect damage present in the building structure under different working environments. The SVM boundary found to shift when the whole class of training data it trained on collected from a building structure with damage in it. It contains layers of ANN kernel functions. In back-propagation networks, adjusting the weight and bias reduces the error between measurement and output. Yang et al. evaluated SVM, self-organizing feature maps, and support vectors for detecting an error. The wavelet transforms used to extract the attributes from the vibration data and raw Noise data. The most accurate method is the hybrid, which combines the SVMs, LVQ (Learning Vector Quantization).Figure-4 represents the full system of the Hybrid RS-SVM \& RS-ANN component, which adjusted, and a calculation proposed for discretization. RST gets the principles and further makes the information vectors having the most basic and essential highlights. This standard base on a decreased arrangement then utilized to prepare the spatial information bases.

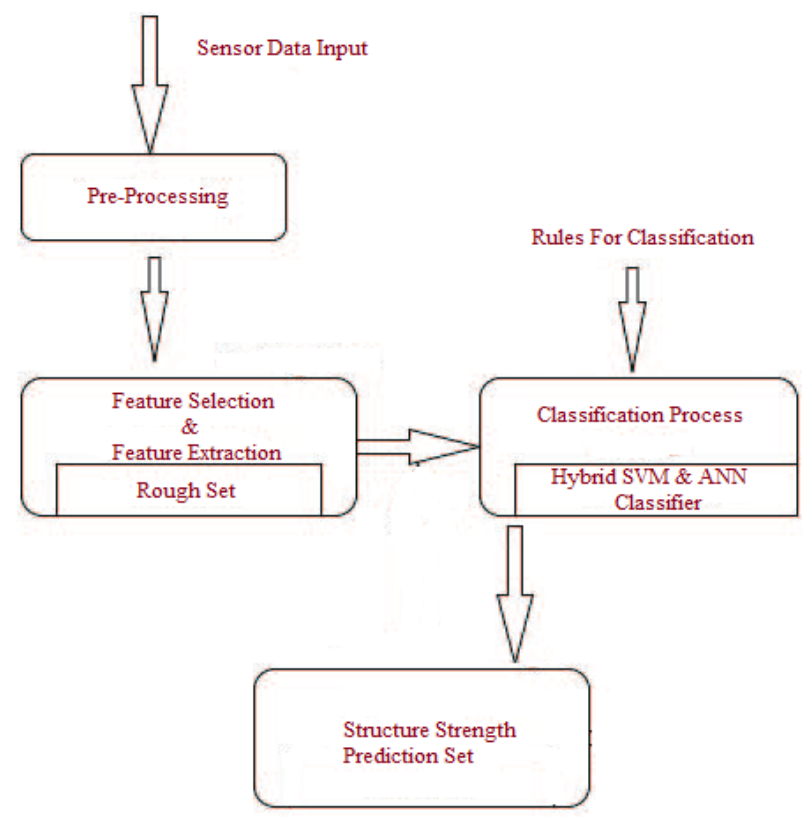

Figure 4: Full prototype flow chart for the hybrid method.

\footnotetext{
Algorithm: 2: RAS (Combined Rough set Artificial neural network Support vector machine) metaheuristic

Step 1:

Gathering data from sensors and create Initial attribute Set $(\mathrm{IaS})$ with $\mathrm{n}$ attributes.
} 


$$
\operatorname{IaS}=\sum_{i=0}^{n} a_{i}
$$

Where ' $a$ ' the attribute names and ' $i$ ' index or instances.

\section{Step 2:}

The Decision System (DS) defined as follows.

$$
D S: F a S=\left(I a S, \operatorname{IaS}_{c} \cup\left\{\mathrm{D}_{a}\right\}\right)
$$

Where $\mathrm{IaS}_{c}$ is a non-empty finite set of attributes, $\forall \mathrm{a} \in \mathrm{IaS}_{c}, a: I a S \rightarrow V_{a}$ the value set of a. is Decision attributes.

The elements are called $\mathrm{IaS}_{c}$ conditional attributes and $\mathrm{FaS}$ are the Final attribute Set.

\section{Step 3:}

For each attribute in $\mathrm{FaS}$ repeat the following step A.

\section{Step 4:}

Step A: Estimate the dependency degree change (DDC).

Stored DDC in FaS.

Step 5:

FaS is input to the SVM model for finding Structural damage (SD) using a hyperplane.

Step 6:

$\mathrm{SD}$ is input to the ANN model for finding Damage Levels (DL).

Step 7:

STOP.

\section{2 result analysis}

The below table 1 shows that Result Analysis of the proposed method.

Table 1: Result Analysis

\begin{tabular}{|c|c|c|}
\hline $\begin{array}{c}\text { S. } \\
\text { NO }\end{array}$ & $\begin{array}{c}\text { METHOD } \\
\text { NAME }\end{array}$ & $\begin{array}{c}\text { ACCURACY } \\
(\%)\end{array}$ \\
\hline 1 & SVM & 85.33 \\
\hline 2 & ANN & 86.93 \\
\hline 3 & RAS & 91.24 \\
\hline
\end{tabular}

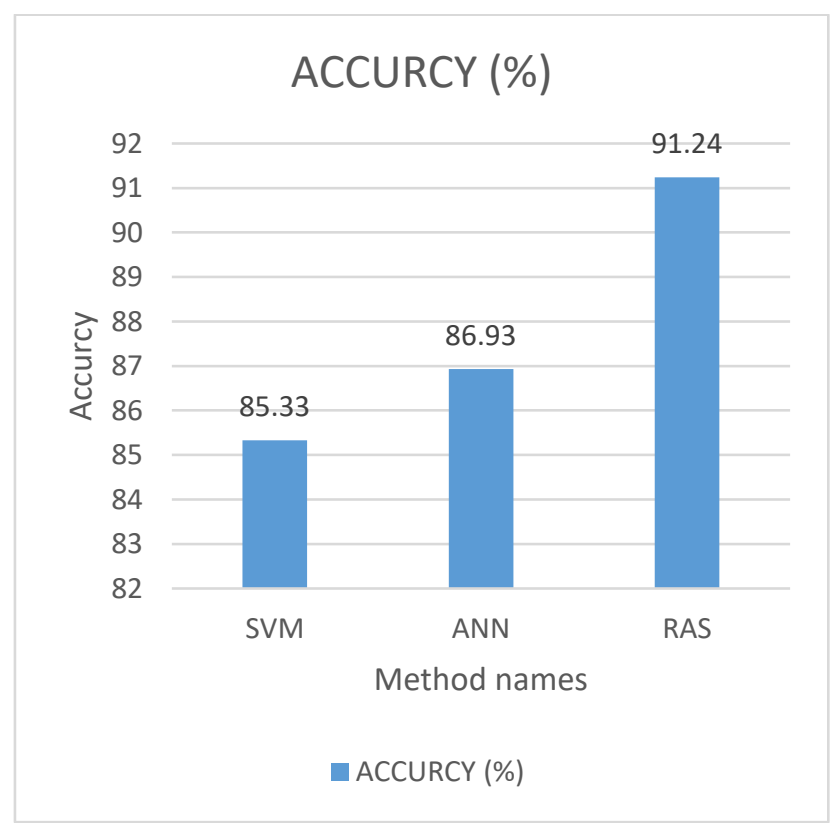




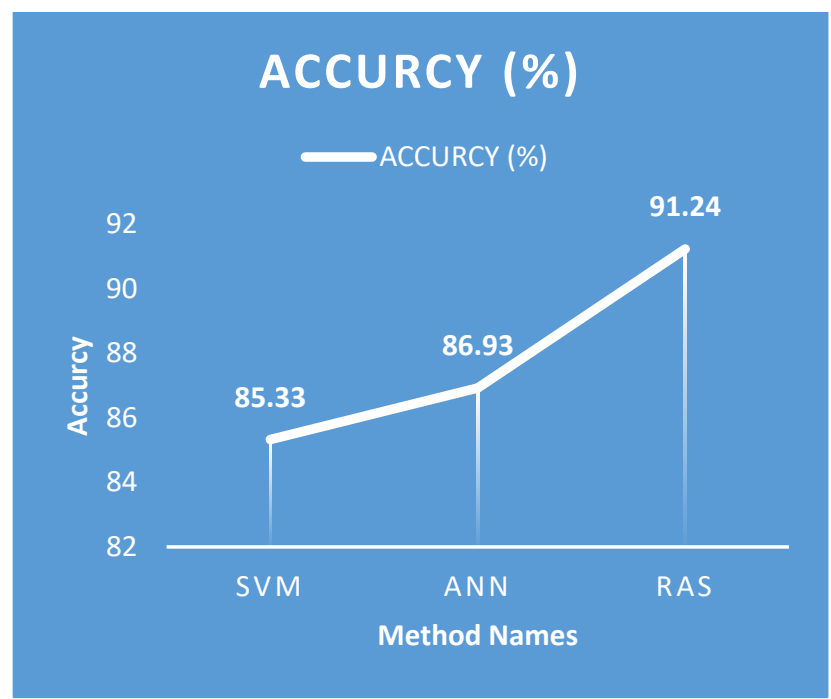

Figure 5: The accuracy of the proposed method.

\section{Conclusion}

Nowadays, the fault status assessment is an essential issue for civil engineering researchers. Structural Strength Monitoring System is an efficient method for predicting the damages using the vibration data gathered from sensors. However, the sensing signal contains noise. In the proposed method, RST used as a pre-processing step for reducing the processing time by eliminating the redundant features and noise in the sensing signal. SVM and ANN combine result analysis section indicate that the hybrid classification algorithm (RAS) accuracy is better than individuals.

\subsection{Future work}

In the proposed method, we use only one preprocessing method like RST, so in future work, use the hybrid pre-processing methods and check the accuracy with the RAS algorithm.An efficient method needs for structural strength monitoring system done by arranging wireless sensors at different parts of the building, number of sensors, power systems for sensors. The data gathered from the sensors arranged into structural order from the unbuilding structured format. Based on the structured building data, the strength of the building assessed. All of the above work done offline. The future work is developing the online methods, places of the sensor, creating the building structured vibration data, types of sensors, and power systems for sensors.

\section{References}

1. C. Nerves and R. Krishnan, "Active control strategies for tall civil building structures," in Proc. IEEE Int. Conf. Ind. Electron., Control, Instrum., 1995, vol. 2, pp. 962-967.

2. K. Chopra, Dynamics of Building structures: Theory and Application to Earthquake Engineering, 2nd ed. Englewood Cliffs, NJ, USA: rentice-Hall,2001.

3. K. Chopra, Dynamics of Building structures: Theory and Application to Earthquake Engineering, 2nd ed. Englewood Cliffs, NJ, and USA: Prentice-Hall, 2001.

4. Link and H. J. von Martens, “Accelerometer identification using shock excitation," Measurement, vol. 35, no. 2, pp. 191-199, Mar. 2004.

5. Adams, R.D., Cawley, P., Pye, C.J. and Stone, B.J.(1978). A vibration technique for non-destructively assessing the integrity of building structures. Journal of Mechanical Engineering Science, 20, 93-100.

6. R. Farrar and Worden, Structural Health Monitoring: A Machine Learning Perspective. Hoboken, NJ, USA: Wiley, 2013.

7. R. Soc. London A Math. Phys. Eng. Sci., vol. 365, no. 1851, pp. 303-315, 2007.

a. Rytter, "Vibrational Based Inspection of Civil Engineering Structures." Dept. of Building Technology and Structural Engineering, Aalborg University, 1993.

8. Doebling, S.W.; Farrar, C.R.; Prime, M.B. A summary review of vibration-based damage identification methods. Shock Vib. Dig. 1998, 30, 91-105.

9. Sohn, H.; Farrar, C.R.; Hemez, F.M.; Shunk, D.D. A Review of Structural Health Monitoring Literature: 1996-2001; Los Alamos National Laboratory: New Mexico, NM, USA, 2004.

10. Carden, E.P.; Fanning, P. Vibration based condition monitoring: A review. Struct. Health Monit. 2004, 3,355-377.

11. Wang, L.; Chan, H.T. "Review of vibration-based damage detection and condition assessment of bridge Structures using structural health monitoring." In Proceedings of the Second Infrastructure Theme 
Postgraduate Conference: Rethinking Sustainable Development: Planning, Engineering, Design and Managing Urban Infrastructure, Brisbane, Australia, and 26 March 2009.

12. Fan, W.; Qiao, P.Z. Vibration-based damage identification methods: A review and comparative study. Struct. Health Monit. 2011, 10, 83-111.

13. Moughty, J.J.; Casas, J.R. Vibration Based Damage Detection Techniques for Small to Medium Span Bridges: A Review and Case Study. In Proceedings of the 8th European Workshop on Structural Health Monitoring (EWSHM 2016), Bilbao, Spain, 5-8 July 2016.

14. Das, S.; Saha, P.; Patro, S.K. Vibration-based damage detection techniques used for health monitoring of structures: A review. J. Civ. Struct. Health Monit. 2016, 6, 477-507.

15. Xuan Kong, Chun-Sheng Cai and Jiexuan Hu "The State-of-the-Art on Framework of Vibration-Based Structural Damage Identification for Decision Making.” Appl. Sci. 2017, 7, 497; doi: 10.3390/app7050497,

16. Li H, Yang H, Hu SLJ (2006) Modal strain energy decomposition method for damage localization in 3D frame structures. J Eng Mech 132(9):941-951

17. Seyedpoor SM (2012) A two stage method for structural damage detection using a modal strain energy based index and particle swarm optimization. Int J Non Linear Mech 47(1):1-8

18. Grande E, Imbimbo MJ (2014) A multi-stage data-fusion procedure for damage detection of linear systems based on modal strain energy. J Civil Struct Health Monit 4(2):107-118

19. Li Y, Wang S, Zhang M, Zheng C (2016) An improved modal strain energy method for damage detection in offshore platform structures. JMSA 15(2):182-192

20. Pal J, Banerjee S (2015) A combined modal strain energy and particle swarm optimization for health monitoring of structures. J Civil Struct Health Monit 5(4):353-363.

21. Ashory MR, Ghasemi-Ghalebahman A, Kokabi MJ (2017) An efficient modal strain energy-based damage detection for laminated composite plates. Adv Compos Mater 27(2):147-162.

22. Adams RD, Cawley P, Pye CJ, Stone BJ (1978) A vibration techniquefor non-destructively assessing the integrity of structures. JMech Eng Sci 20(2):93-100

23. Hassiotis S, Jeong GD (1995) Identification of stiffness reductionsusing natural frequencies. J Eng Mech 121(10):1106-1113

24. Messina A, Williams EJ, Contursi T (1998) Structural damagedetection by a sensitivity and statisticalbased method. J SoundVib 216(5):791-808

25. Maity D, Tripathy RR (2005) Damage assessment of structuresfrom changes in natural frequencies using genetic algorithm. Struct Eng Mech 19(1):21-42

26. Beena P, Ganguli R (2011) Structural damage detection usingfuzzy cognitive maps and hebbian learning. Appl Soft Comput11(1):1014-1020

27. [28 detection in beam-like structures usinggenetic algorithms. Appl Soft Comput 8(2):1150-1160

28. Kao CY, Chen XZ, Jan JC (2016) Locating damage to structuresusing incomplete measurements. J Civil Struct Health Monit6(5):817-838

29. Ding ZH, Huang M, Lu ZR (2016) Structural damage detectionusing artificial bee colony algorithm with hybrid search strategy.Swarm Evol Comput 28:1-13

30. Gharechahi A, Ketabdari MJ (2017) A novel method for selectingmeasurement points in structural model updating and damagedetection. J Civil Struct Health Monit 7(4):471-482

31. Ercolani GD, Felix DH, Ortega NF (2018) Crack detection inprestressed concrete structures by measuring their natural frequencies.J Civil Struct Health Monit 8(4):661-671.

32. Lee U, Shin J (2002) A frequency response function-based structural damage identification method. Comput Struct 80(2):117-132

33. Mohan SC, Maiti DK, Maity D (2013) Structural damage assessment using FRF employing particle swarm optimization. ApplMath Comput 219(20):10387-10400.

34. Stutz LT, Castello DA, Rochinha FA (2005) A flexibility based continuum damage identification approach. J Sound Vib279(3):641-667

35. Li J, Wu B, Zeng QC, Lim CW (2010) A generalized flexibility matrix based approach for structural damage detection. J Sound Vib 329(22):4583-4587

36. [37 damage detection using sparse sensors installation by optimization procedure based on the modal flexibility matrix. J Sound Vib 381:65-82

37. Chandrashekhar M, Ganguli R (2009) Structural damage detection using modal curvature and fuzzy logic. Struct Health Monit 8(4):267-282.

38. Ulrike Dackermann, Jianchun Li and Bijan Samali "Damage Index Method for Damage Identification utilising Artificial Neural Networks" Eng.Struct.In Press (2009).

39. [40 index method using neural network ensembles". Eng. Struct. (2008) In Press

40. G. W. Housner, L. A. Bergman, T. K. Caughey et al., "Structural control: past, present, and future, "Journal of Engineering Mechanics, vol. 123, no. 9, pp. 897-971, 1997. 
41. Dackermann, U., Li, J. \& Samali, B. 2010. "Boundary damage identification of a two-storey framed structure utilising frequency response functions and artificial neural networks", Proceedings of the 10th International Conference on Motion and Vibration Control, Tokyo, Japan, Paper \#1A12, (published on CD).

42. K. T. Park, S. H. Kim, H. S. Park, and K. W. Lee, "The determination of bridge displacement using measured acceleration," Engineering Building structures, vol. 27, no. 3, pp. 371-378, 2005.

43. Kim, J.T. and Stubbs, N. (2003). Crack detection in beam-type building structures using frequency data. Journal of Sound and Vibration, 259, 145-160.

a. Liang, R.Y., Choy, F.K. and Hu, J.L. (1991). Detection of cracks in beam building structures using measurements of natural frequencies. Journal of the Franklin Institute-Engineering and Applied Mathematics, 328, 505-518.

44. Chinchalkar, S. (2001). Determination of crack location in beams using natural frequencies. Journal of Sound and Vibration, 247, 417-429.

45. F. Moschas and S. Stiros, "Measurement of the dynamic displacements and of the modal frequencies of a short-span pedestrian bridge using GPS and an accelerometer," Engineering Building structures, vol. 33, no. 1, pp. 10-17, 2011

46. E. Forstner and H.Wenzel, "The application of data mining in bridge monitoring projects: exploiting time series data of structural health monitoring," in Proceedings of the $22^{\text {nd }}$ International Workshop on Database and Expert Systems Applications, 2011.

47. J. Yang, J. B. Li, and G. Lin, "A simple approach to integration of acceleration data for dynamic soilbuilding structure interaction analysis," Soil Dyn. Earthquake Eng., vol. 26, no. 8, pp. 725-734, Aug. 2006.

48. K. P. Bennett and E. J. Bredensteiner, Geometry in Learning, Geometry at Work, Mathematical Association of America, 2000, Edited by C. Gorini.

49. H. Hothu and A. Mita, "Damage Detection Method Using Support Vector Machine and First Three Natural Frequencies for Shear Structures,” vol. 2013, no. June, pp. 104-112, 2013.

50. Rao, M. Vishnu Vardhana, and Aparna Chaparala. "A Building Damage Classification Framework for Feature Subset Selection using Rough Set with Mutual Information." Solid State Technology (2020): 498509.

51. Chang, C.-C. And C. J. Lin (2001). LIBSVM: a library for support vector machines. Software available at http://www.csie.ntu.edu.tw/ cjlin/libsvm.

52. Zhong Zuowei, Mu Lili (2011) “The Integrated Methodology of Rough Set Theory and Artificial Neural Network for Safety Assessment on Construction Sites" Third International Conference on Intelligent Human-Machine Systems and Cybernetics.

53. Majumdar A, Maiti DK, Maity D (2012) Damage assessment of truss structures from changes in natural frequencies using ant colony optimization. Appl Math Comput 218(19):9759-9772.

54. Nanda B, Maity D, Maiti DK (2014) Crack assessment in frame structures using modal data and unified particle swarm optimization technique. Adv Struct Eng 17(5):747-766

55. Kang F, Li JJ, Xu Q (2012) Damage detection based on improved particle swarm optimization using vibration data. Appl Soft Comput 12(8):2329-2335

56. Wei Z, Liu J, Lu Z (2018) Structural damage detection using improved particle swarm optimization. Inverse Probl Sci Eng 26(6):792-810

57. Li J, Zhang X, Xing J (2015) Optimal sensor placement for longspancable-stayed bridge using a novel particle swarm optimizationalgorithm. J Civil Struct Health Monit 5(5):677-685

58. Ding ZH, Huang M, Lu ZR (2016) Structural damage detection using artificial bee colony algorithm with hybrid search strategy. Swarm Evol Comput 28:1-13.

59. Chou JH, Ghaboussi J (2001) Genetic algorithm in structural damage detection. Comput Struct 79(14):1335-1353

60. Laier JE, Morales JDV (2009) Improved genetic algorithm for structural damage detection. In: Yuan Y, Cui J, Mang HA (eds) Computational structural engineering. Springer, Dordrecht, pp 833-839

61. Silva M, Santos A, Figueiredo E, Santos R, Sales C, Costa JCWA(2016) A novel unsupervised approach based on a genetic algorithmfor structural damage detection in bridges. Eng Appl Artif Intell 52:168-180.

62. Meruane V, Heylen W (2011) An hybrid real genetic algorithm to detect structural damage using modal properties. Mech Syst Signal Process 25(5):1559-1573.

63. Wang FL, Chan THT, Thambiratnam DP (2013) Damage diagnosisfor complex steel truss bridges using multi-layer geneticalgorithm. J Civil Struct Health Monit 3(2):117-127.

64. Tsou P, Shen MHH (1994) Structural damage detection and identification using neural networks. AIAA J 32(1):176-183

65. Neves AC, González I, Leander J (2017) Structural health monitoring of bridges: a model-free ANN-based approach to damage detection. J Civil Struct Health Monit 7(5):689-702. 
66. E. Forstner and H. Wenzel, "The application of data mining in bridge monitoring projects: Exploiting time series data of structural health monitoring," in Proc. 22nd Int. Workshop Database Expert Syst. Appl., 2011, pp. 297-301.

67. Link and H. J. von Martens, “Accelerometer identification using shock excitation," Measurement, vol. 35, no. 2, pp. 191-199, Mar. 2004.

68. M. E. Mavroforakis, M. Sdralis, and S. Theodoridis, "A geometric nearest point algorithm for the efficient solution of the SVM classification task," IEEE Transactions on Neural Networks, vol. 18, no. 5,pp. 15451549, 2007.

69. Maity, D. and Tripathy, R.R. (2005). Damage assessment of building structures from changes in natural frequencies using genetic algorithm. Structural Engineering and Mechanics, 19, 21-42.

70. R. Collobert, F. Sinz, J. Weston, and L. Bottou, "Trading convexity for scalability," in Proceedings of the 23rd International Conference on Machine Learning (ICML '06), pp. 201-208, Pittsburgh, Pa, USA, June 2006.

71. S. Kalyani, "Classification and assessment of power system security using multiclass SVM," IEEE Transactions on Systems, Man, and Cybernetics C, vol. 41, no. 5, pp. 753-758, 2011.

72. S. Thenozhi, W. Yu, and R. Garrido, "A novel numerical integrator for velocity and position estimation," Trans. Inst. Meas. Control, vol. 35, no. 6, pp. 824-833, Aug. 2013.

73. X. Meng, A. H. Dodson, and G. W. Roberts, "Detecting bridge dynamics with GPS and triaxial accelerometers," Engineering Building structures, vol. 29, no. 11, pp. 3178-3184, 2007.

74. M.Vishnu vardhana rao, Aparna Chaparala, "Analysis of classification technique for Prediction of Damages levels in Building-Structures", International Journal of Advanced Science and Technology ,Vol. 29, No. 05, (2020), pp. 822-842.

75. K. Srivastava, K. S. Patnaik, L. Bhambhu'Data Classification: A Rough - SVM Approach" Contemporary Engineering Sciences, Vol. 3, 2010, no. 2, $77-86$ 